\title{
强冲击作用下舰船结构毁伤的三维无网格 SPH-RKPM方法数值模拟
}

\author{
彭玉祥, 张阿漫", 薛冰, 礼绍博 \\ 哈尔滨工程大学船舶工程学院, 哈尔滨 150001 \\ *联系人, E-mail: zhangaman@hrbeu.edu.cn \\ 收稿日期: 2020-11-09; 接受日期: 2021-03-08; 网络出版日期: 2021-06-15 \\ 国家自然科学基金(编号: 51925904, 51909042)资助项目
}

\begin{abstract}
摘要 强冲击载荷会严重威胁舰船生命力, 对于冲击作用下舰船结构毁伤的精确预报是舰船防护设计和提高舰 船生命力的重要环节. 考虑到传统有网格算法在结构断裂模拟上遇到的挑战，本文在前期工作的基础上，建立了 强冲击载荷作用下结构毁伤的完全无网格模型与计算方法, 采用光滑粒子流体动力学 (Smoothed Particle Hydrodynamics, SPH)方法计算水下爆炸载荷, 基于重构核粒子方法(Reproducing Kernel Particle Method, RKPM)建立结 构接触、断裂计算模型. 依据法通量方法, 耦合RKPM和SPH构建了完全无网格流固耦合三维模型与计算方法, 通 过多个复杂算例充分验证了该数值计算模型的有效性.进一步开发了强冲击作用下舰船结构毁伤模拟程序, 提高 了程序的鲁棒性. 该程序作为模块已融入到具有完全自主知识产权的FSLAB工业软件之中. 与传统网格计算方法 相比, 本文数值模型在处理结构断裂、损伤等方面有优势, 可为舰船防护结构设计提供基础性技术支撑.
\end{abstract}

关键词＼cjkstart结构毁伤, 强冲击, 光滑粒子流体动力学方法(SPH), 重构核粒子方法(RKPM)

PACS: 47.40.Rs, 07.05.Tp, 79.20.Ap, 61.20.Ja, 46.70.-p, 62.50.Ef

\section{1 引言}

舰船防护结构设计是舰船生命力研究的重要内 容 ${ }^{[1,2]}$, 准确预报舰船结构在强冲击载荷作用下的毁伤 特性能为舰船防护结构设计提供基础性技术支撑，从 而有效提高舰船生命力。在强冲击载荷如舰船碰 撞 ${ }^{[3,4]}$ 、弹头对于舷侧的打击 ${ }^{[5]}$ 、近场水下爆炸 ${ }^{[6-8]}$ 等 $^{\text {等 }}$ 的作用下, 结构会产生较大的塑性变形, 甚至发生损伤 断裂或出现破口，涉及结构大变形以及多介质的非线
性耦合作用.

对于这种极端载荷作用下复杂舰船结构毁伤的预 报, 理论研究的局限性很大, 难以给出准确、全面的结 构毁伤公式或者解析解，通常仅能得到简单结构的毁 伤估算公式，例如水下爆炸作用下的平板破口估算公 式 ${ }^{[9]}$. 目前在该领域的研究中, 主要采用实验研究和数 值研究. 其中实验研究是最直观可信的方案, 但是由于 实验中舰船结构会发生不可逆的毁伤，因此相关研究 的成本极高. 缩比实验虽然能够节约成本，但采用缩

引用格式: 彭玉祥, 张阿漫, 薛冰, 等. 强冲击作用下舰船结构毁伤的三维无网格SPH-RKPM方法数值模拟. 中国科学: 物理学 力学天文学, 2021, 51: 124614 Peng Y X, Zhang A M, Xue B, et al. Numerical investigation of ship structure damage subject to strong impact using a 3D meshless SPH-RKPM method (in Chinese). Sci Sin-Phys Mech Astron, 2021, 51: 124614, doi: 10.1360/SSPMA-2020-0403 
比模型进行实验很难完全满足相似性定律 ${ }^{[10]}$. 相较于 理论和实验研究, 数值研究不仅能模拟冲击作用下复 杂结构的毁伤, 而且成本较低 ${ }^{[11]}$. 随着数值计算理论 的不断完善，它被越来越多地应用到了舰船结构毁伤 的模拟当中 ${ }^{[12]}$.

目前数值算法主要分为有网格算法和无网格算法 两大类, 其中有网格算法的应用更加广泛, 例如结构分 析领域的有限元方法(Finite Element Method, FEM) ${ }^{[13]}$ 和计算流体领域的有限体积方法(Finite Volume Method, FVM $)^{[14]}$. 然而传统有网格算法在强冲击作用下的 结构毁伤研究中面临着挑战：基于网格的有限元方法 难以模拟裂纹间断，而基于欧拉表述的算法需要额外 算法来追踪复杂物质界面 ${ }^{[15-17]}$. 无网格算法由于不依 赖网格, 可以自由改变粒子间的连接关系, 能方便地模 拟裂纹间断. 同时, 基于拉格朗日表述的无网格算法能 自动追踪物质界面，极大简化了多相流数值模拟的难 度. 鉴于此, 无网格算法成为了计算力学领域的研究 热点 ${ }^{[18,19]}$.

在计算流体研究领域当中应用最广的无网格算法 是光滑粒子流体动力学方法(Smoothed Particle Hydrodynamics, SPH), 它被广泛应用到了自由表面流 ${ }^{[20,21]}$ 、 水下爆炸动力学 ${ }^{[22-24]}$ 、结构砰击入水 ${ }^{[25,26]}$ 等问题的 研究当中. 本文采用 $\mathrm{SPH}$ 方法来计算水下爆炸载荷, 同 时基于重构核粒子方法(Reproducing Kernel Particle Method, RKPM)建立舰船复杂加筋壳结构动力学计算 模型. 由于本文RKPM结构算法仅采用一层粒子对壳 体进行离散, 因此基于法通量方法(Normal-Flux Method ${ }^{[27]}$ 将结构考虑为流体的动边界, 在 SPH控制方程中 考虑边界截断项的影响, 实现结构对流体的作用. 而流 体对结构的影响则认为是加载在结构上的压力载荷, 其大小通过结构附近流场压力近似得到.

本文简要给出了RKPM加筋壳弹塑性断裂的基本 理论, 并对RKPM壳体接触算法做了阐述; 之后给出了 依据法通量方法建立的SPH-RKPM流固耦合三维数值 计算模型; 开展了一系列典型数值算例研究, 并将计算 结果与有限元方法对比, 在之前研究的基础之上, 进一 步验证了SPH-RKPM无网格数值计算模型在强冲击作 用下舰船等复杂结构毁伤预报中的有效性，开发了相 应的计算程序，已作为模块融入到了具有完全自主知 识产权的流固耦合FSLAB软件之中.

\section{2 基于重构核粒子法的壳结构数值计算 模型}

重构核粒子法是Liu等人 ${ }^{[28]}$ 通过提高传统的SPH 方法中核函数一致性, 并基于Galerkin方法建立弱形式 控制方程得到的. 其通过选取不同的基函数, 能够使修 正后的型函数满足任意阶数的一致性，进而极大提高 了算法的计算精度. 目前RKPM方法已经被广泛应用 到了结构大变形响应的模拟当中 ${ }^{[29-31]}$. 本文基于 RKPM建立复杂加筋壳计算模型，仅需在壳参考面上 布置一层粒子来对空间壳体进行离散 ${ }^{[32]}$. 根据Mindlin-Reissner壳理论，假定: (1) 壳的纤维矢量变形之后 仍保持为直线; (2) 变形过程中壳体板厚保持不变 ${ }^{[33]}$. 这样可以将三维的壳体退化为参考面和初始与参考面 垂直的纤维矢量组成的集合. 通过重构核函数对壳单 元物理量离散, 在此基础上, 依据虚功率原理(Principle of Virtual Power)获得RKPM壳单元离散形式的控制方 程如下:

$$
\begin{aligned}
& \int_{S_{0}} \rho_{0} h N_{I} \mathrm{~d} S \dot{\mathbf{v}}_{I}(\mathbf{x}, t) \\
& =\int_{S_{0}} \rho_{0} h N_{I} \mathbf{b} \mathrm{d} S_{0}+\int_{\Gamma_{0}^{t}} N_{I} \overline{\mathbf{t}}^{0} \mathrm{~d} \Gamma_{0}^{t}-\int_{V_{0}} \frac{\partial N_{I}}{\partial \mathbf{X}} \mathbf{P} \mathrm{d} V,
\end{aligned}
$$

$$
\begin{aligned}
& \frac{h^{2}}{12} \int_{S_{0}} \rho_{0} h N_{I} \mathrm{~d} S \dot{\boldsymbol{\omega}}_{I} \\
& =\int_{\Gamma_{0}^{t}} \frac{h}{2} N_{I} \cdot \mathbf{y}_{I} \times \overline{\mathbf{t}}^{0} \mathrm{~d} \Gamma_{0}^{t}+\int_{V_{0}} \frac{h}{2} \frac{\partial \widetilde{N}}{\partial \mathbf{X}} \mathbf{P} \times \mathbf{y}_{I} \mathrm{~d} V,
\end{aligned}
$$

式中, $\rho_{0}$ 表示壳体初始密度, $N_{I}$ 表示RKPM型函数, b表 示单位质量体积力, $\overline{\mathbf{t}}^{0}$ 表示壳体受到的边界力, $\mathbf{P}$ 表示 Piola-Kirchhoff应力张量(第一类别), $\widetilde{N}_{I}=\zeta N_{I}$, 其中 $\zeta$ 为壳厚度方向的参数坐标, $y_{I}$ 表示 $I$ 粒子的纤维矢量, $V_{0}$ 表示 $I$ 粒子支持域的壳体, $S_{0}$ 表示对应的壳体参考面, $\Gamma_{0}^{t}$ 表示牵引力边界. 通过求解上面的控制方程就可以 实现对壳体动力响应的模拟. 不过在强冲击作用下, 壳体会发生弹塑性大变形，甚至会发生断裂，同时对 于舰船复杂加筋壳结构, 壳体不同部分还会发生碰撞, 需建立无网格的接触算法以及损伤断裂计算模型.

\subsection{RKPM壳体接触计算模型}

RKPM壳体接触算法分为两部分：一是壳体接触 
检测算法; 二是接触力计算模型. 接触检测即判断壳体 不同部分何时接触，需要搜索每个应力点周围的节点， 我们采用链表搜索算法 ${ }^{[12]}$. 计算中, 首先搜索应力点 附近的壳体节点，当二者之间的相对位置矢量满足如 下关系时认为应力点和节点存在接触:

$\left\|\mathbf{x}_{J I} \mathbf{e}_{l 3}^{l}\right\|<h$,

$\sqrt{\left(\mathbf{x}_{J I} \mathbf{e}_{l 1}^{l}\right)^{2}+\left(\mathbf{x}_{J I} \mathbf{e}_{l 2}^{l}\right)^{2}}<\lambda a$,

上式中 $\mathbf{x}_{J I}=\mathbf{x}_{J}-\mathbf{x}_{I}$, 其中 $I$ 为应力点编号, $J$ 为节点编号. $\mathbf{e}_{l 1}^{l}, \mathbf{e}_{l 2}^{l}$ 和 $\mathbf{e}_{l 3}^{l}$ 代表壳应力点处三个方向的基矢量, 上标 $l$ 表示薄层坐标系(lamina coordinate system). $h$ 表示壳体 厚度, $\lambda$ 为常数, 用来控制接触区域大小, 在本研究中, $\lambda$ 取为 $0.75 . a$ 表示光滑长度, 一般取为粒子间距. 上式 表明, 当两个切向距离很近的粒子, 其垂向距离小于壳 体厚度时认为两部分壳体产生接触. 需要注意的是, 在 计算过程中，需排除组成应力点所属积分单元的壳 体节点，因为这些节点和应力点虽然满足上面的接触 判据，但是它们属于相同的壳体微元，不应计入相互 接触.

当壳体结构产生接触, 这时需给壳体节点施加接 触力, 来防止非物理穿透现象. 这里忽略摩擦力的影 响, 则应力点 $I$ 和节点 $J$ 之间的接触力可以表示为

$\mathbf{f}_{J I}=\kappa_{n} \cdot \mathbf{e}_{I 3}^{1} \cdot \operatorname{sign}\left(\mathbf{x}_{J I} \cdot \mathbf{e}_{I 3}^{1}\right)$,

式中, $\kappa_{n}=0.01 K S_{I} \cdot\left(h-d_{n}\right) / h$, 表示法向接触力大 小，其中 $K$ 表示材料体积模量. $S_{I}$ 表示应力点 $I$ 的面 积. $d_{n}$ 表示应力点和节点之间的法向距离. 符号函数 $\operatorname{sign}\left(\mathbf{x}_{J I} \cdot \mathbf{e}_{I 3}^{1}\right)$ 表征节点位于应力点的哪一边. 当节点与 多个应力点接触时, 节点受到接触力的取值, 通过与 这些应力点之间接触力的平均得到，而且不同侧节点 受到应力点接触力方向相反. 耦合接触检测及接触力 算法, 即可建立RKPM壳接触分析数值模型.

\section{2 弹塑性损伤本构模型}

弹塑性本构模型的建立是舰船结构断裂毁伤模拟 的基础. 在数值模拟当中, 首先通过结构变形计算得到 应力点处厚度方向各积分点的变形率张量 ${ }^{[34]}$. 这里考 虑舰船结构材料为次弹性材料, 采用Hughes-Winget方 法 $^{[35]}$ 可以得到当前时间步的有效试应力为 $\bar{\tau}_{n+1}^{\text {trial }}=\mathbf{Q}_{n+1} \cdot \bar{\tau}_{n} \cdot \mathbf{Q}_{n+1}^{\mathrm{T}}+\mathbf{C}_{e l}^{\nabla J}: \mathbf{d}_{n+1} \cdot \Delta t$,

其中, $\mathbf{Q}_{n+1}=\mathbf{I}_{3}+\left(\mathbf{I}_{3}-\mathbf{w}_{n+1} / 2\right)^{-1} \mathbf{w}_{n+1}, \mathbf{I}_{3}$ 表示秩为 3 的单 位矩阵, $\mathbf{w}_{n+1}$ 为速度梯度反对称部分. $\mathbf{C}_{e l}^{\nabla J}$ 为弹性模量 张量, $\mathbf{d}_{n+1}$ 表示当前变形率张量. 有效试应力定义为 $\bar{\tau}=\tau /(1-D)$, 其中 $D$ 表示损伤变量. 在求得试应力之 后，将其带入到Mises屈服准则中，计算屈服函数 $\phi_{n+1}{ }^{\text {trial }}$ 的值. 若 $\phi_{n+1}^{\text {trial }}<0$, 则说明材料应力状态仍在屈服面内, 当前步的试应力即为材料的真实应力. 反之, 则说明材 料应力状态在屈服面外, 需要更新材料屈服面, 同时将 应力状态返回到新的屈服面上，具体算法参考文献 [34]中的描述. 同时如果材料的等效塑性应变满足了 以下判据, 还要对材料的损伤变量进行更新:

$f_{D}\left(\epsilon_{\mathrm{p}}\right)=\left[\frac{2}{3}(1+v)+3(1-2 v)\left(\frac{\tau_{\mathrm{m}}}{\tau_{\mathrm{eq}}}\right)^{2}\right] \epsilon_{\mathrm{p}}-\epsilon_{\mathrm{p}}^{D}$,

其中 $v$ 表示材料泊松比, $\tau_{\mathrm{m}}$ 和 $\tau_{\mathrm{eq}}$ 分别表示静水压力和等

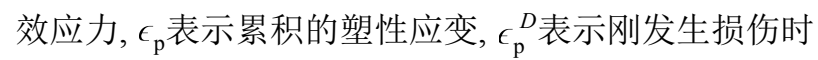
的应变. 若 $f_{D}\left(\epsilon_{\mathrm{p}}\right)<0$ 表明材料没有损伤，可以直接采 用塑性模型来确定材料的应力状态. 反之则表明材料 发生了损伤，需要通过计入及损伤的塑性返回算法来 确定材料的应力状态. 损伤演化方程 ${ }^{[36]}$ 为

$\dot{D}=\frac{D_{\mathrm{c}}}{\epsilon_{\mathrm{p}}^{R}-\epsilon_{\mathrm{p}}^{D}}\left[\frac{2}{3}(1+v)+3(1-2 v)\left(\frac{\tau_{\mathrm{m}}}{\tau_{\mathrm{eq}}}\right)^{2}\right] \dot{\epsilon_{\mathrm{p}}}$,

上式中 $\epsilon_{\mathrm{p}}^{R}, D_{\mathrm{c}}$ 分别为材料断裂应变、损伤阈值.

\subsection{RKPM裂纹拓展计算模型}

从宏观角度看, 结构中裂纹拓展就相当于在其内 部产生了新的自由边界, 从而导致连续的物理场出现 间断. 传统的有限元方法需要通过拓展有限元法 ${ }^{[37]}$ 或 相场法 ${ }^{[38,39]}$ 来模拟这种间断，且已经广泛应用到了断 裂的模拟当中. 不过目前针对复杂壳结构大变形延性 断裂的研究还不十分成熟, 且算法较为复杂. 无网格 方法可以自由改变粒子间的连接关系，因此可以方便 地模拟裂纹间断 ${ }^{[40]}$. 同时相较于有网格算法, 无网格 算法在断裂破片的模拟方面存在一定优势.

本文通过应力点损伤变量值来建立裂纹萌生算 法. 具体地, 首先通过计算出应力点处壳厚度方向积分 
点上损伤变量的数值, 在此基础上, 当这些积分点损伤 变量的平均值大于断裂损伤阈值 $D_{\mathrm{c}}$ 时，积分点全部损 伤 ${ }^{[34]}$, 进而形成新的裂纹尖端, 表示如下:

$\frac{\sum_{i=1}^{n} D_{i}}{n}>D_{\mathrm{c}}$,

上式中 $n$ 为壳体厚度方向高斯积分点数量, $D_{i}$ 为厚度方 向各积分点处损伤变量的取值. 当上述裂纹萌生判据 满足后，即形成了新的裂纹尖端，将新老裂纹尖端连 接便形成了裂纹拓展路径. 随着新的裂纹拓展路径形 成，裂纹表面切割粒子支持域。这里裂纹拓展路径可 视为不透明的墙，处于裂纹路径两侧的粒子相互不可 见，也即没有连接关系, 粒子支持域也被截断了, 根据 粒子新的支持域即可更新插值函数 ${ }^{[34]}$ 从而实现裂纹 间断的模拟.

\section{SPH-RKPM流固耦合三维计算模型}

无网格SPH方法由于能自动追踪界面同时不受网 格畸变困扰, 被广泛应用到爆炸毁伤 ${ }^{[41]}$ 、冲击碰撞 ${ }^{[42]}$ 等问题的研究中，本文选取SPH方法来模拟水下爆炸 载荷. SPH方法基于拉格朗日表述, 采用粒子填充计算 域, 这些粒子携带流场物理量并占据一定空间, 粒子的 物理量可通过其支持域内粒子的物理量近似表达为

$$
f\left(\mathbf{x}_{I}\right)=\sum_{J} f\left(\mathbf{x}_{J}\right) W\left(\mathbf{x}_{J}-\mathbf{x}_{I}\right) \frac{m_{J}}{\rho_{J}},
$$

上式中 $W_{I J}$ 表示核函数, $m_{J}$ 表示粒子质量, $\rho_{J}$ 代表粒子 密度. 而对于函数的梯度, SPH的离散表达式为

$$
\begin{aligned}
\nabla f\left(\mathbf{x}_{I}\right)= & \sum_{J} f\left(x_{J}\right) \cdot \nabla_{I} W\left(x_{J}-x_{I}\right) \frac{m_{J}}{\rho_{J}} \\
& +\sum_{K} f\left(x_{K}\right) W\left(x_{K}-x_{I}\right) \mathbf{n}_{K} S_{K},
\end{aligned}
$$

其中 $K$ 代表结构粒子, $\mathbf{n}_{K}$ 表示边界法线, 指向流体域外, $S_{K}$ 为壳结构粒子的面积. 在传统SPH方法当中, 由于核 函数的紧支性, 忽略了式(11)当中的边界积分项. 在本 文的流固耦合研究中, 将结构看作流体域的动边界. 考 虑到流固耦合界面会使附近流体粒子支持域截断，式 (11)梯度算子中的边界积分项需要考虑进来. 所谓的 法通量方法就是通过考虑式(11)边界积分项来实现结 构壁面的不可穿透条件. 修正之后的流体控制方程表 示为

$$
\begin{aligned}
\frac{D \rho_{I}}{D t}= & -\rho_{I} \sum_{J} \mathbf{v}_{I J} \cdot \nabla_{I} W_{I J} \frac{m_{J}}{\rho_{J}}-\rho_{I} \sum_{K} \mathbf{v}_{I K} W_{I K} \mathbf{n}_{K} S_{K}, \\
\frac{D \mathbf{v}_{I}}{D t}= & -\frac{1}{\rho} \sum_{J} \bar{p}_{I J} \cdot \nabla_{I} W_{I J} \frac{m_{J}}{\rho_{J}}+\alpha c_{0} h \frac{\rho_{0}}{\rho_{I}} \sum_{J} \Pi_{I J} \nabla_{I} W_{I J} \frac{m_{J}}{\rho_{J}} \\
& -\frac{1}{\rho} \sum_{K} \bar{p}_{I K} W_{I K} W_{I K} \mathbf{n}_{K} S_{K}, \\
\frac{D e_{I}}{D t}= & -\frac{p_{I}}{\rho_{I}} \sum_{J} \mathbf{v}_{I J} \nabla_{I} W_{I J} \frac{m_{J}}{\rho_{J}}+\frac{1}{2} \alpha c_{0} h \frac{\rho_{0}}{\rho_{I}} \sum_{J} \Pi_{I J}\left(\mathbf{v}_{J}-\mathbf{v}_{I}\right) \\
& \cdot \nabla_{I} W_{I J} \frac{m_{J}}{\rho_{J}}-\frac{p_{I}}{\rho_{I}} \sum_{K} \mathbf{v}_{I K} W_{I K} \mathbf{n}_{K} S_{K},
\end{aligned}
$$

上式中 $K$ 为结构粒子编号, $I$ 和 $J$ 表示流体粒子编号. $p$, $\rho, v, e$ 分别表示压力、密度、速度以及内能. $c_{0}$ 为初始 声速, $\nabla_{I}$ 为梯度算子. $\mathbf{n}_{K}$ 和 $\mathbf{x}_{K}$ 分别表示结构应力点 $K$ 处 的法向以及结构应力点的空间坐标, $\alpha$ 为人工黏性和人 工热度系数, $\Pi_{I J}$ 为人工黏性项. 流体对结构的作用, 可 看作加载在壳体表面压力载荷，其载荷大小由周围流 场压力近似，将压力载荷带入到结构控制方程中的外 力项中即可求解结构的动力响应. 通过以上公式的求 解, 从而本节建立了SPH-RKPM流固耦合三维计算 模型.

\section{4 数值算例}

本节通过几个数值算例对本文壳体弹塑性接触计 算模型，以及完全无网格流固耦合三维计算模型的有 效性进行验证. 计算中使用双线性RKPM型函数, 同时 采用减缩积分, 即在壳参考面内的各个积分域中仅放 置一个应力点, 剪切自锁现象可得以消除.

\section{1 弹头冲击作用下舰船舷侧舱段的毁伤}

在弹头高速冲击作用下，船舶结构会发生极大的 变形甚至出现破口，对舰船内部舱室结构和设备的安 全造成很大威胁，严重影响舰船生命力。本节采用 RKPM对弹头高速冲击作用下舰船舷侧舱段的毁伤进 行模拟, 计算模型示意图如图1所示, 弹头初始速度大 小为 $200 \mathrm{~m} / \mathrm{s}$. 舱段模型长为 $1.5 \mathrm{~m}$, 最大宽度为 $0.567 \mathrm{~m}$, 最小宽度为 $0.4 \mathrm{~m}$, 高为 $0.71 \mathrm{~m}$. 弹头为直径 $0.08 \mathrm{~m}$ 、总 长 $0.3 \mathrm{~m}$ 的球头弹头. 弹头和舱段采用相同的材料, 密 度为 $\rho=7800 \mathrm{~kg} / \mathrm{m}^{3}$, 杨氏模量 $E=2.1 \times 10^{11} \mathrm{~Pa}$, 泊松 比 $v=0.3$, 初始屈服应力设为 $\sigma_{y 0}=390 \mathrm{Mpa}$. 依据线性硬 


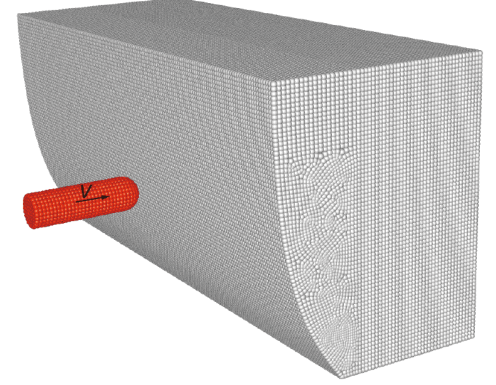

图 1 (网络版彩图)高速撞击下的舰船舷侧防御结构: 问题 描述.

Figure 1 (Color online) Warship broadside defensive structure subjected to high speed impact: Problem description.

化模型, 硬化模量设为 $E_{\mathrm{p}}=1305 \mathrm{MPa}$. 材料损伤演化由 方程(8)得到, 损伤阈值的参数设置为 $D_{\mathrm{c}}=0.4$, 临界损伤

(a)

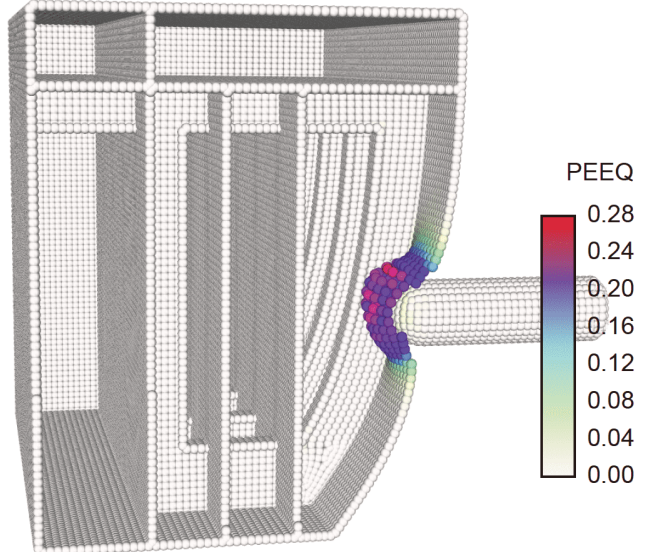

(c)

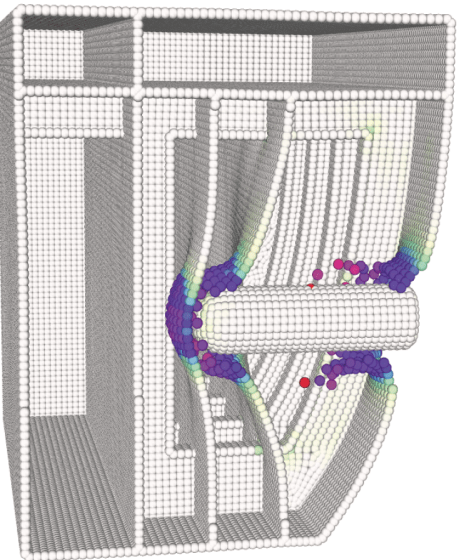

应变 $\epsilon_{\mathrm{p}}{ }^{D}=0.16$, 断裂应变 $\epsilon_{\mathrm{p}}{ }^{R}=0.28$. 材料应变率效应依据 Cowper-Symonds模型得到，考虑应变率强化效应的屈 服应力表示为 $\bar{\sigma}_{y}=\left[1+\left(\dot{\epsilon}_{\mathrm{eq}} / C\right)^{1 / p}\right] \sigma_{y}$, 其中 $\dot{\epsilon}_{\mathrm{eq}}$ 表示等效 塑性应变率, 其中的材料常数设置为 $C=6 \times 10^{-4}$, $p=50.8$. 计算中平均粒子间距设为 $0.01 \mathrm{~m}$, 舷侧舱段粒 子总数为 96595, 弹体粒子总数为 794, 时间步长设置为 $1.0 \times 10^{-7} \mathrm{~s}$.

图2给出了几个典型时刻舷侧舱段的变形构型, 并 绘制出了结构等效应力云图. 从图中可以看出, 计算初 始 $t=0.3 \mathrm{~ms}$ 时随着弹头的冲击, 舷侧外板发生了局部凹 陷，并且与弹头接触区域周边的粒子等效塑性应变达 到了断裂应变, 出现了冲塞断裂. 之后在 $t=1.0 \mathrm{~ms}$ 时, 弹头穿透舷侧外板, 并刚刚要和内层舱壁碰撞. 由于 舱段结构的阻挡, 弹头速度减小, 当 $t=2.0 \mathrm{~ms}$ 时弹头与 (b)

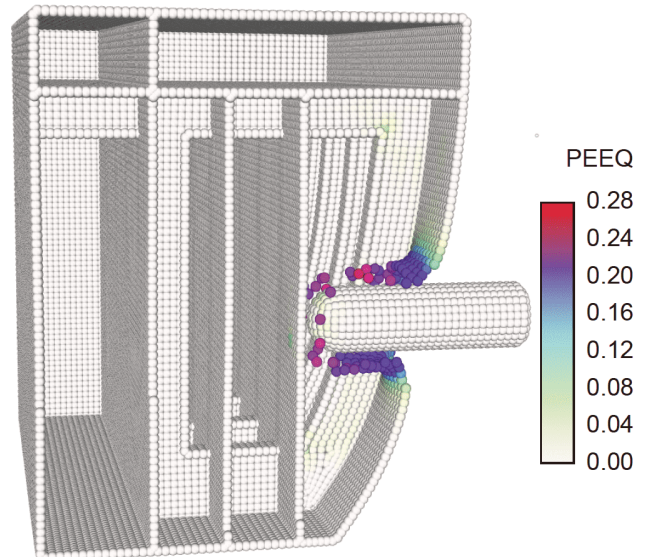

(d)
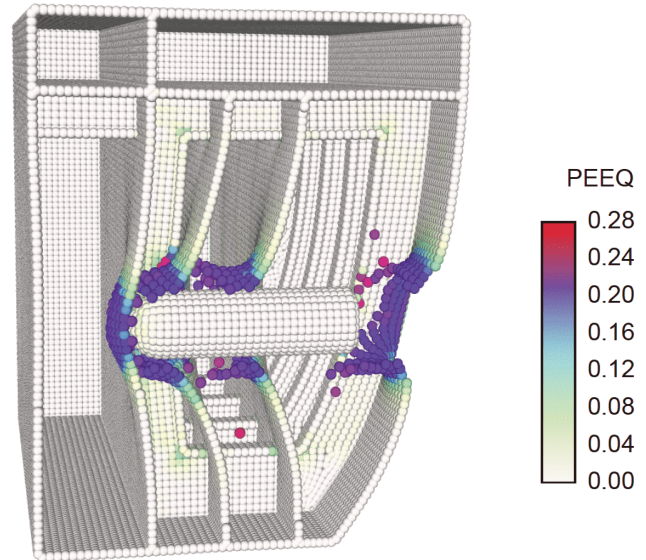

图 2 (网络版彩图) 几个典型时刻的舷侧舱段等效塑性应变云图及变形构型. (a) $t=0.3 \mathrm{~ms}$; (b) $t=1.0 \mathrm{~ms}$; (c) $t=2.0 \mathrm{~ms}$; (d) $t=$ $4.0 \mathrm{~ms}$

Figure 2 (Color online) Contours of the equivalent plastic strain on the deformed configuration of the shipboard at several typical times. (a) $t=$ $0.3 \mathrm{~ms}$; (b) $t=1.0 \mathrm{~ms}$; (c) $t=2.0 \mathrm{~ms}$; (d) $t=4.0 \mathrm{~ms}$. 
第二层舱壁碰撞，两层内部舱壁均发生了极大的塑性 变形. 最后在 $t=4.0 \mathrm{~ms}$ 时内部主防护舱壁也发生了较 大塑性变形，防护结构的阻碍也使得弹头速度下降到 了 $10 \mathrm{~m} / \mathrm{s}$ 以内. 在图3中我们绘制出了弹头速度的时历 曲线，并将RKPM计算结果与ABAQUS软件中的FEM 计算结果进行了对比，从图中可以看出，本文计算结 果与软件计算结果吻合较好，计算模型和方法的有效 性得到了验证。

\section{2 水下爆炸载荷作用下舰船的折断分析}

我们对压力波作用下的舰船弯折过程进行了模 拟，计算模型如图4所示，舰船底部受到了球形压力波 的作用, 压力载荷的加载面用红色粒子表示. 在我们之 前的工作中 ${ }^{[43]}$ ，对该模型在近场水下爆炸作用下的局 部毁伤特性进行了模拟，而这里主要关注中远场水下 爆炸载荷对舰船的整体毁伤。计算中，加载表面的压 力载荷通过下面的公式进行计算:

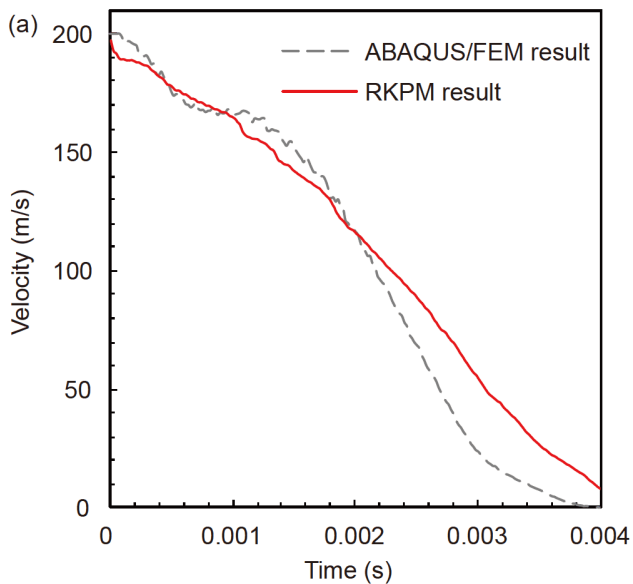

$p_{I}=p_{t}\left(\frac{8}{\left\|\mathbf{X}_{I}-\mathbf{X}_{c}\right\|}\right)^{1.13}$

其中 $X_{c}$ 是球形压力波源的坐标，波源位于船体中部正 下方 $8 \mathrm{~m}$ 处, $X_{I}$ 为压力加载面上应力点 $I$ 的初始坐标, $p_{t}$ 表示冲击波和气泡联合产生的随时间变化的压力, 通过Geers-Hunter模型 ${ }^{[44]}$ 进行计算, 如图5所示. 在建立 几何模型的过程中，只考虑了主要舱壁和大的加强筋， 忽略了小的型材. 计算中将平均的粒子间距设为 $0.4 \mathrm{~m}$, 共采用了 123990 个粒子对舰船进行离散. 船体外壳厚 度设为 $0.012 \mathrm{~m}$, 其他板厚均设为 $0.007 \mathrm{~m}$. 采用固定的 时间步长, 且有 $\Delta t=1.0 \times 10^{-5} \mathrm{~s}$. 在强冲击问题中, 应变 率效应对结果有显著影响. 在本研究中, 材料应变率效 应依据Cowper-Symonds模型得到，舰船具体的材料参 数参考文献[43].

在图6中给出了几个典型时刻的舰船变形构型, 并 在其中显示出了船体的Mises等效应力云图. 从中可得

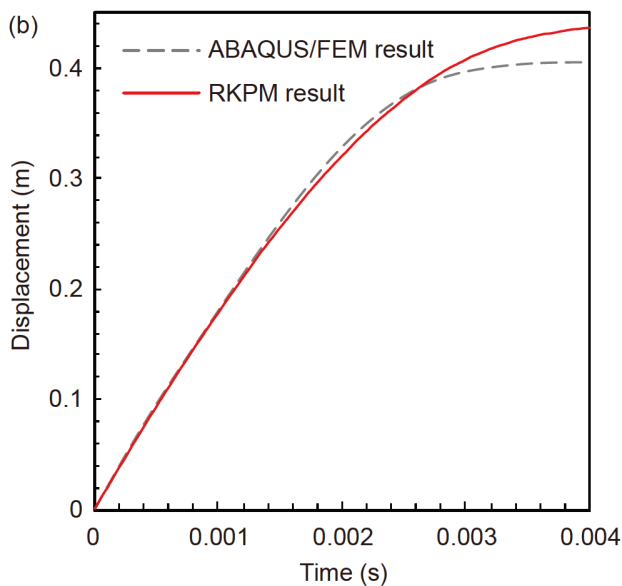

图 3 (网络版彩图) RKPM计算结果和ABAQUS/FEM计算结果对比. (a) 弹头速度时历曲线; (b) 弹头位移时历曲线

Figure 3 (Color online) Comparison between the RKPM result and ABAQUS/FEM result. (a) Velocity history of the warhead; (b) displacement history of the warhead.

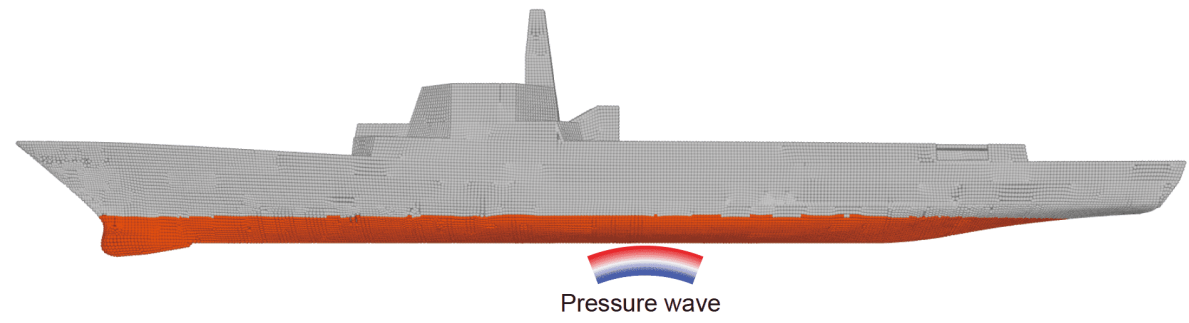

图 4 (网络版彩图)压力波作用下的舰船弯曲, 压力载荷的加载面用红色粒子标出

Figure 4 (Color online) Bending of the warship under pressure wave, in which the loaded surface is denoted by the red particles. 


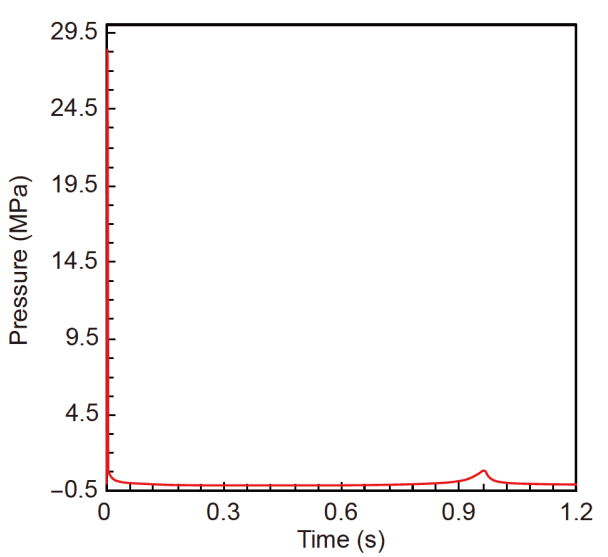

图 5 (网络版彩图)舰船离爆点最近点的压力载荷时历曲线 Figure 5 (Color online) Pressure history of the nearest point on the ship from the blast point.

到, 当计算开始之后, 船体中部在压力波的作用下向上 拱起, 船体进入中拱状态. 之后, 由于压力波大小小于 大气压力, 相当于有一个向下的吸引力加载在船体上, 导致船体中部开始向下变形. 由于持续时间较长, 导致 船体产生明显的中垂弯曲, 最终导致船体中部折断, 如 图6(d)所示. 同时, 我们可以将舰船结构简化为船体梁, 采用文献 $[45,46]$ 中提出的无量纲参数能够对冲击作用 下的舰船折断进行理论分析，由于船体梁是变截面的，
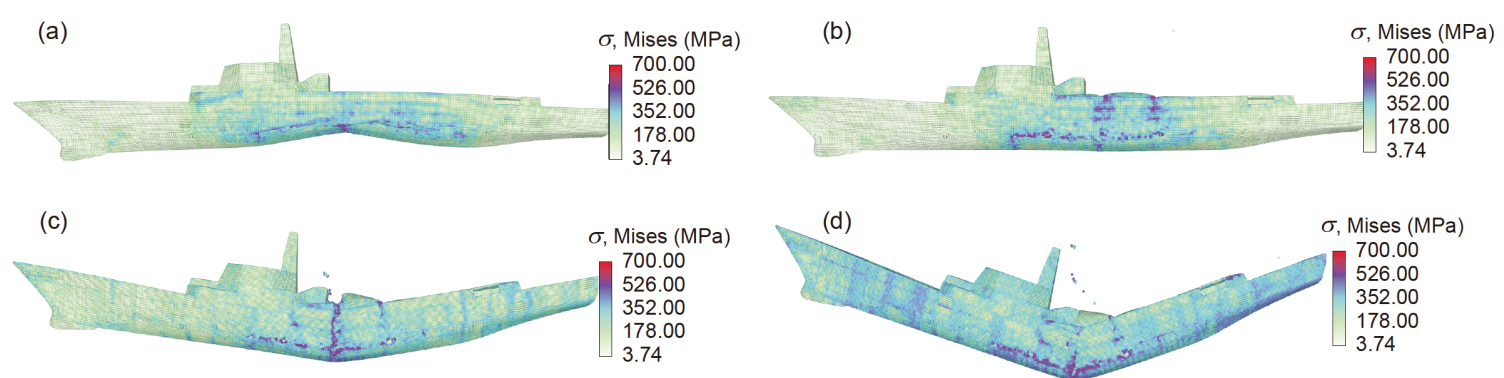

所以原始的无量纲参数需要做相应的修正.

由于船体中垂弯曲变形极大, 导致中部许多粒子 均发生了损伤，形成了一个损伤区，如图7中红色虚线 标记区域所示. 同时, 从图中可以看出, 船体甲板和外 板均发生了极大的变形，在近场水下爆炸下舰船的毁 伤主要是船中底部产生了局部破口 ${ }^{[43]}$, 对比可以发现 近场水下爆炸和中远场水下爆炸对舰船的毁伤模式有 很大区别. 此外, 图7将本文RKPM计算结果和ABAQUS软件计算结果进行了对比, 可以看到两者计算得 到的舰船最终的变形构型吻合良好. 在数值模拟当中, 没有发现不稳定现象, 验证了本文RKPM结构算法在 复杂舰船结构毁伤模拟当中的适用性和鲁棒性.

\section{3 水下爆炸载荷作用下平板的毁伤}

本节对水下近场接触爆炸作用下的平板毁伤进行 模拟, 并用LS-DYNA中的有限元方法对相同的模型进 行计算, 对比验证SPH-RKPM数值模型的有效性. 计算 中水域尺寸设置为 $12 \mathrm{~m} \times 12.4 \mathrm{~m} \times 6 \mathrm{~m}$ ，尺寸为 $12 \mathrm{~m} \times$ $12.4 \mathrm{~m}$ 的平板背空在水域正上方, 平板厚度为 $0.01 \mathrm{~m}$. 计算工况为, 当量 $8.5 \mathrm{~kg}$ 的 Trinitrotoluene药包在平板 中心正下方 $0.25 \mathrm{~m}$ 处爆炸, 采用Jones-Wilkins-Lee状态 方程来求解爆炸气体压力，水的压力则采用Mie-Gru-

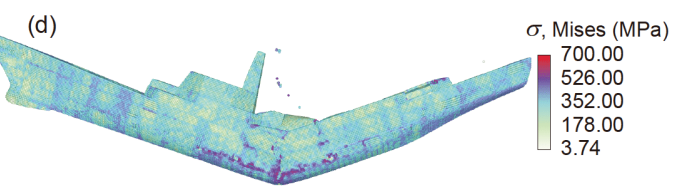

图 6 (网络版彩图) 几个典型时刻的舰船变形构型. (a) $t=0.15 \mathrm{~s}$; (b) $t=0.4 \mathrm{~s}$; (c) $t=0.6 \mathrm{~s}$; (d) $t=0.8 \mathrm{~s}$

Figure 6 (Color online) Deformation configuration of the ship at several typical times. (a) $t=0.15 \mathrm{~s}$; (b) $t=0.4 \mathrm{~s}$; (c) $t=0.6 \mathrm{~s}$; (d) $t=0.8 \mathrm{~s}$.
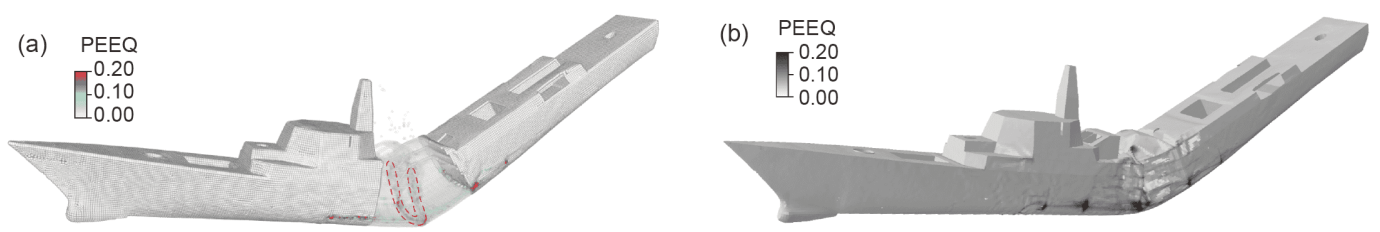

图 7 (网络版彩图) RKPM计算得到的舰船最终变形构型与ABAQUS/FEM计算结果对比. (a) RKPM结果; (b) ABAQUS/FEM 结果

Figure 7 (Color online) Compare the final deformed configuration obtained by RKPM with the ABAQUS/FEM result. (a) RKPM result; (b) ABAQUS/FEM result. 
neisen状态方程计算得到，其中状态方程参数参考Liu 等人 ${ }^{[23]}$ 的研究.

平板的材料为低合金钢，密度 $\rho=7800 \mathrm{~kg} / \mathrm{m}^{3}$, 杨 氏模量 $E=2.1 \times 10^{11} \mathrm{~Pa}$, 泊松比 $v=0.3$, 初始屈服应 力 $\sigma_{y 0}=390 \mathrm{MPa}$, 依据线性硬化模型, 硬化模量设为 $E_{\mathrm{p}}=$ $600 \mathrm{MPa}$. 材料损伤过程通过方程(8)得到，损伤阈值 为 $D_{\mathrm{c}}=0.32$, 临界损伤应变为 $\epsilon_{\mathrm{p}}^{D}=0.16$, 断裂应变为 $\epsilon_{\mathrm{p}}^{R}=$ 0.28. 材料应变率效应依据Cowper-Symonds模型获得, 材料常数取 $C=40, p=5$. 计算中水和结构的初始粒子间 距均设置为 $0.1 \mathrm{~m}, \mathrm{TNT}$ 的初始粒子间距设置为 $0.05 \mathrm{~m}$, 计算中时间步长设置为 $5.0 \times 10^{-6} \mathrm{~s}$.

图8中给出了 $t=10 \mathrm{~ms}$ 时流场的压力云图以及平板 的变形构型, 并在平板变形构型上给出了结构的Mises 等效应力云图，其中爆炸气体用灰色粒子表示. 从图8 中可发现炸药起爆时就造成了平板的局部变形，并且 炸药接触部位处的结构应力较大，同时由于炸药爆轰 过程极为剧烈, 少量爆炸气体粒子穿透了平板.

在图9中给出了模拟过程中几个典型时刻的平板 变形构型, 并绘出了平板的等效塑性应变云图. 从图中 可以看出, 计算初始平板在爆炸载荷作用下, 平板中心
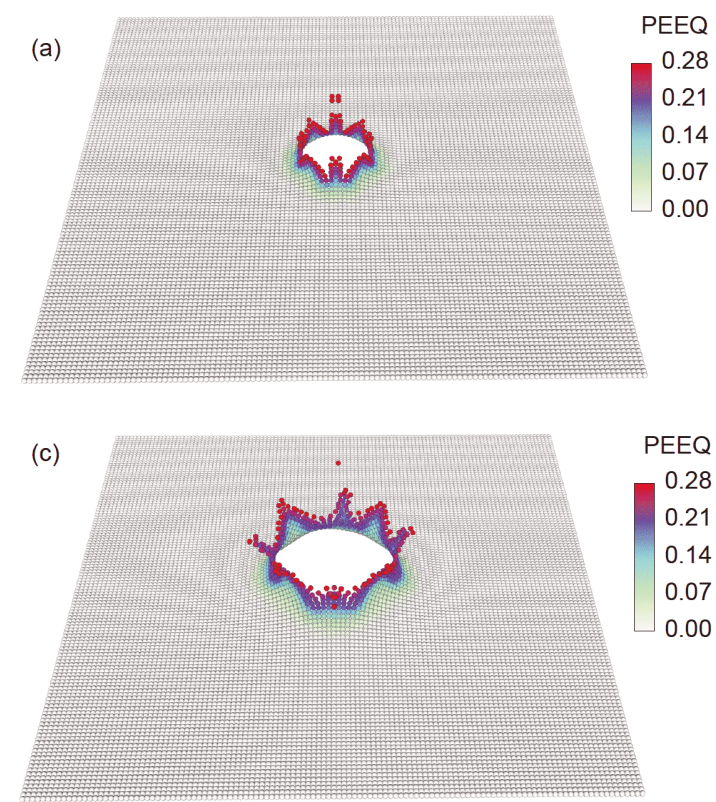

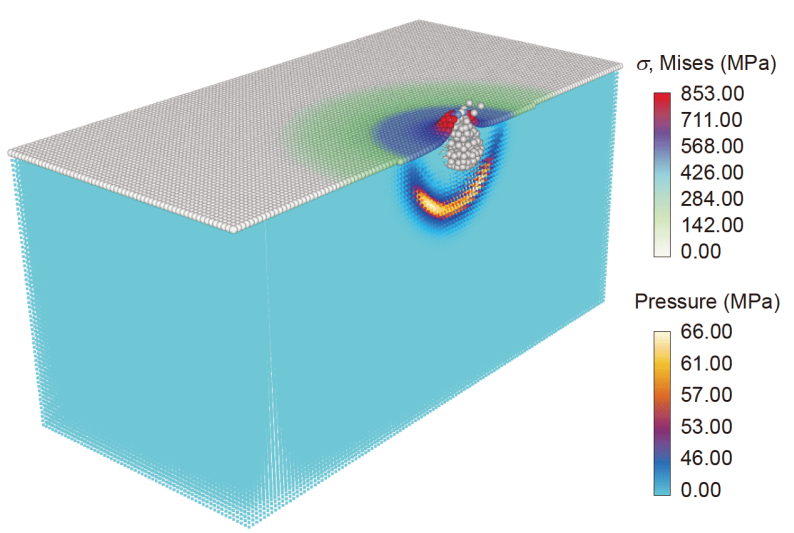

图 8 (网络版彩图)平板接触爆炸 $t=10 \mathrm{~ms}$ 时刻流场的压力 云图以及平板Mises等效应力云图

Figure 8 (Color online) Contour of fluid pressure and Mises equivalent stress of the plate subjected to contact underwater explosion at $t=10 \mathrm{~ms}$.

发生局部凸起变形, 并且出现了花瓣状断裂. 之后爆炸 产物继续膨胀，同时花瓣状破口在膨胀流体作用下继 续扩大，平板局部凸起的面积也进一步变大．在图 10 中将RKPM计算得到的平板最终破口尺寸同有限元结 果进行了对比, 可以看出, SPH-RKPM计算得到的破口 大小稍小于有限元的计算结果. 本文数值结果和有限
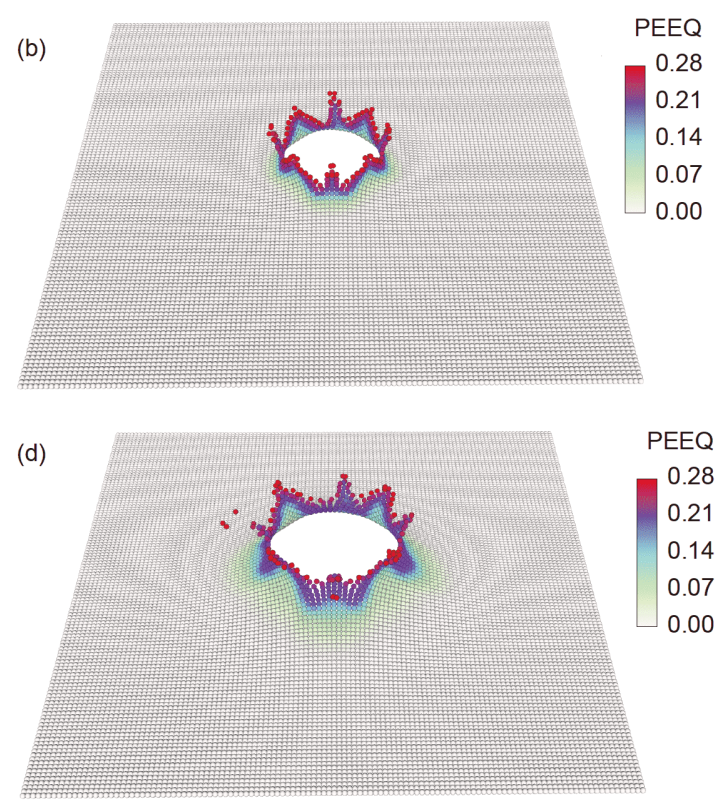

图 9 (网络版彩图)水下近场接触爆炸下平板结构几个典型时刻的等效塑性应变云图. (a) $t=25 \mathrm{~ms}$; (b) $t=50 \mathrm{ms;} \mathrm{(c)} t=$ $100 \mathrm{~ms}$; (d) $t=200 \mathrm{~ms}$

Figure 9 (Color online) Contour of equivalent plastic strain of the plate subjected to contact underwater explosion at several typical times. (a) $t=$ $25 \mathrm{~ms}$; (b) $t=50 \mathrm{~ms}$; (c) $t=100 \mathrm{~ms}$; (d) $t=200 \mathrm{~ms}$. 

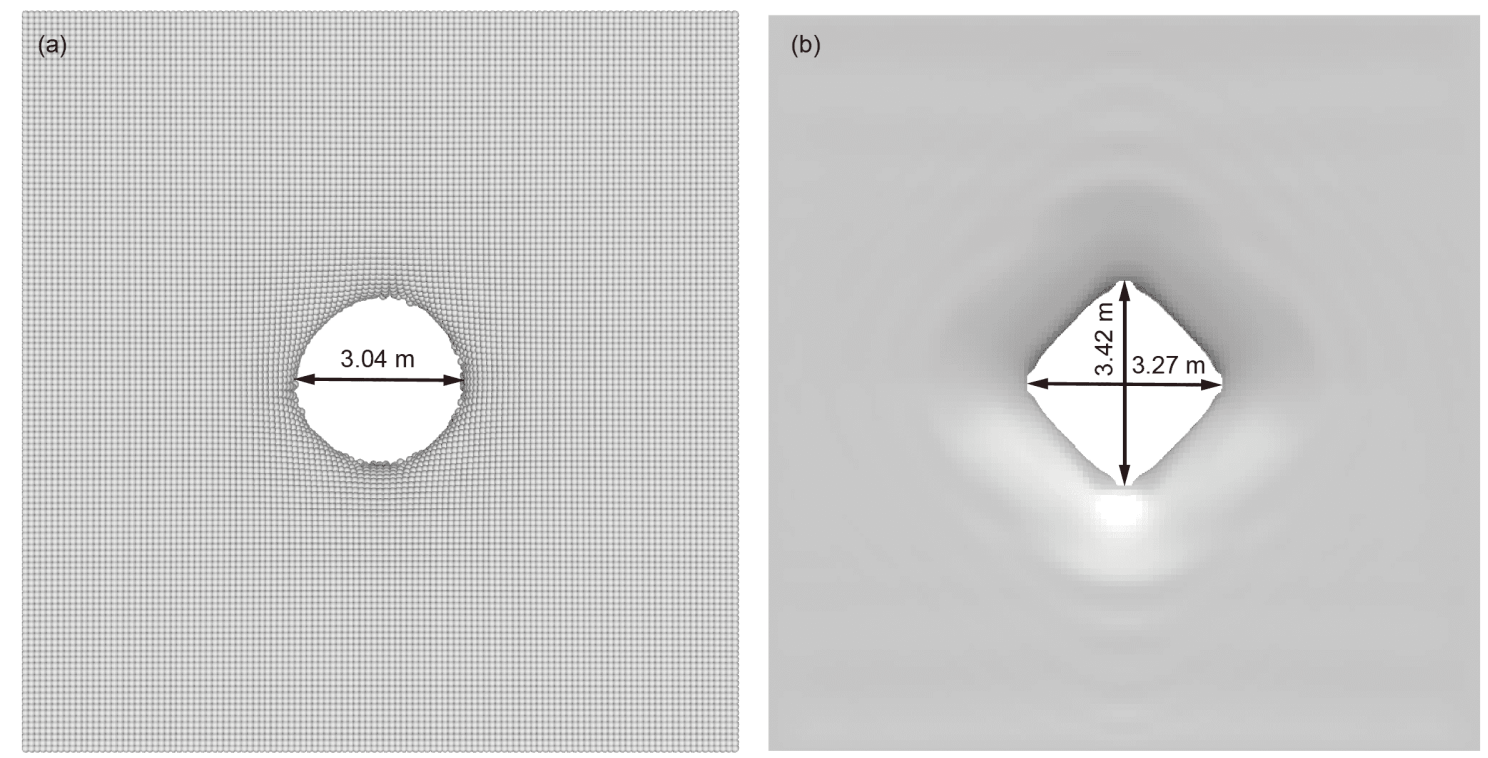

图 10 平板水下接触爆炸SPH-RKPM与LS-DYNA/FEM计算结果对比. (a) SPH-RKPM计算结果; (b) LS-DYNA/FEM计算结果 Figure 10 Comparison of SPH-RKPM and LS-DYNA/FEM results of the plate subjected to underwater contact explosion. (a) SPH-RKPM result; (b) LS-DYNA/FEM result.

元结果的差异可能是由于SPH方法的拉格朗日特性, 导致在模拟后期爆炸产物粒子分布变得稀疏，流体载 荷不能充分传递到结构上.

\section{4 水下爆炸载荷作用下双层底的毁伤}

在前期研究的基础上，本节开展舰船双层底在水 下接触爆炸作用下的毁伤效应数值模拟，以验证耦合 SPH-RKPM数值计算模型在复杂舰船结构毁伤模拟中 的有效性和适用性. 计算模型示意图如图11所示, 船体 板厚度均为 $6 \mathrm{~mm}$ ，矩形加强筋厚度均为 $4 \mathrm{~mm}$. 采用

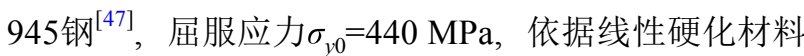
模型, 硬化模量设为 $E_{\mathrm{p}}=1000 \mathrm{MPa}$, 应变率效应由Cow-

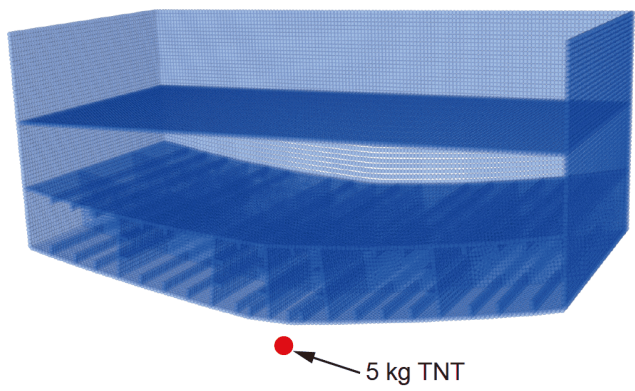

图 11 (网络版彩图)双层底舱段水下接触爆炸工况示意图 Figure 11 (Color online) Schematic diagram of the double bottom compartment subjected to underwater contact explosion.
per-Symonds模型得到，其中相关常数设为 $C=9870$, $p=2.43$. 材料损伤演化由方程(8)得到，损伤阈值设 为 $D_{\mathrm{c}}=0.32$, 临界损伤应变 $\epsilon_{\mathrm{p}}^{D}=0.16$, 断裂应变 $\epsilon_{\mathrm{p}}^{R}=0.28$. 计算中结构的初始平均粒子间距为 $0.04 \mathrm{~m}$ ，水的初始 粒子间距为 $0.04 \mathrm{~m}$, TNT的初始粒子间距为 $0.02 \mathrm{~m}$, 计 算时间步长设置为 $5 \times 10^{-7} \mathrm{~s}$.

图 12 给出了 $0.35 \mathrm{~ms}$ 时的流场压力云图、爆炸气 体形态以及结构变形构型，并在结构粒子上绘制出了 Mises等效应力云图. 可以看出，炸药水下起爆之后， 在很短的时间内船底外板就由于爆炸冲击波的冲击及 爆炸产物的膨胀而产生局部大变形，炸药正上方船体 外板隆起破裂. 由于爆炸产物膨胀速度极快，少量炸 药粒子与船体结构之间发生了穿透.

图13给出了几个典型时刻双层底结构的等效塑性 应变云图. 可以看出, 在 $t=0.2 \mathrm{~ms}$ 时刻, 爆点正上方的双 层底外板应变达到断裂应变值出现破口, 由于内部加 强结构的作用, 裂纹呈现十字状. 在 $t=1.5 \mathrm{~ms}$ 时, 船底 外板在爆轰产物的作用下破口面积增大，与内部板架 连接处出现较明显的塑性应变. 在 $t=4.5 \mathrm{~ms}$ 时, 破口和 塑性变形面积进一步增大，但是内部板架结构仍具有 承载能力. 在 $t=8 \mathrm{~ms}$ 时，破口面积达到最大，破口中心 处的内部板架基本全部达到断裂应变, 进而失效, 破口 


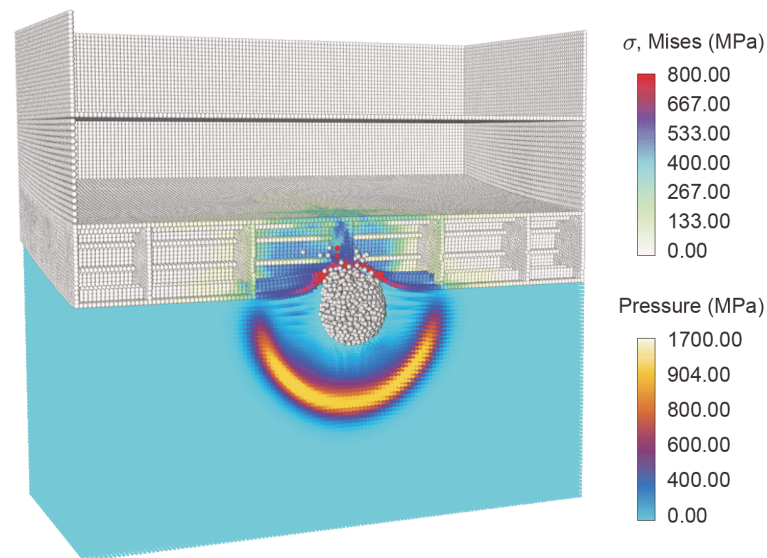

图 12 (网络版彩图)接触爆炸双层底在 $t=0.35 \mathrm{~ms}$ 时刻流场 压力云图及舱段Mises等效应力云图

Figure 12 (Color online) Contour of fluid pressure and Mises equivalent stress of the double bottom compartment subjected to contact underwater explosion at $t=0.35 \mathrm{~ms}$.

(a)

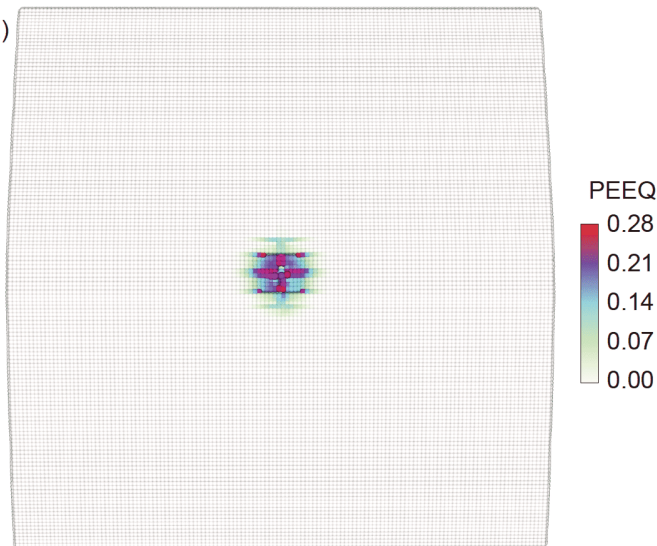

(c)

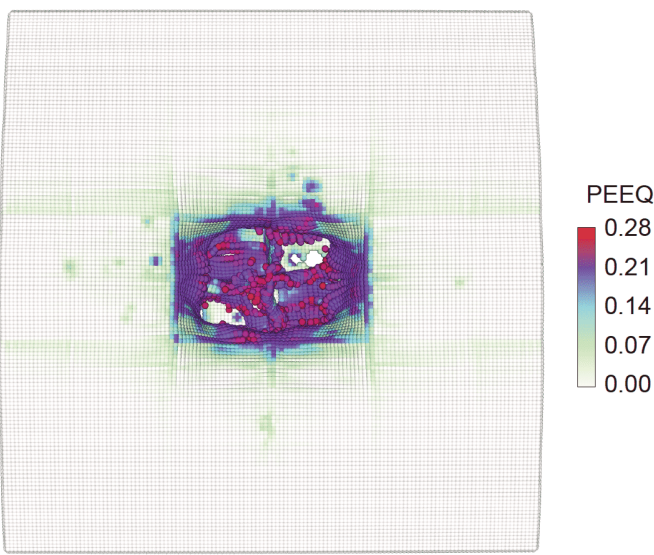

的纵横长度被限制在两倍板架间距内.

图14对比了SPH-RKPM与FEM的计算得到的船 底外板破口。 SPH-RKPM计算得到的破口纵向长 $1.27 \mathrm{~m}$, 横向长 $0.88 \mathrm{~m}, \mathrm{FEM}$ 计算得到的破口纵向长 $1.38 \mathrm{~m}$, 横向长 $0.89 \mathrm{~m}$. 相比于 4.3 节中水下接触爆炸下 平板毁伤，本节SPH-RKPM的结果和FEM结果不论是 形状还是尺寸均更为吻合. 分析原因应该是在本节中, 炸药半径虽为前文的 0.83 倍，但炸药粒子间距却为前 文的 0.25 倍，也即采用了更多的粒子对TNT进行了离 散，后期爆炸气体与结构的作用更充分，从而能在一 定程度上提高后期数值的计算精度.

\section{5 结论}

在强冲击，例如高速碰撞、爆炸冲击等载荷作用

(b)

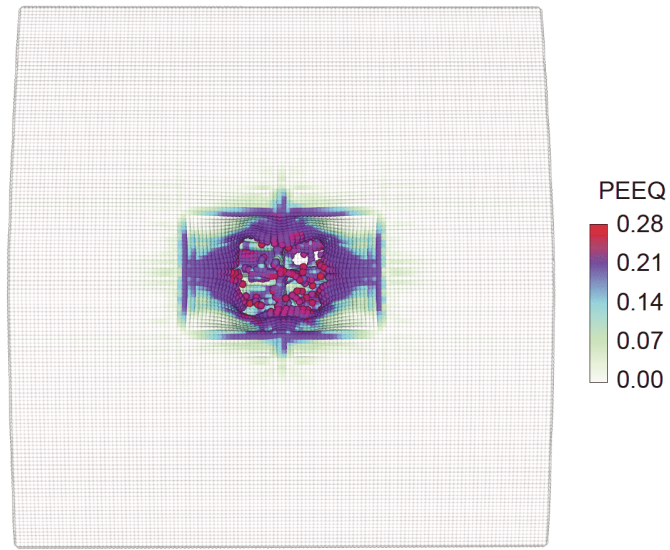

(d)

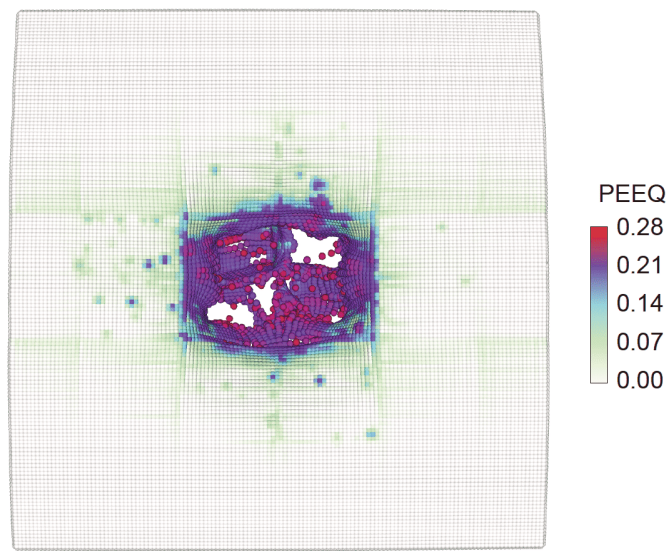

图 13 (网络版彩图)水下接触爆炸载荷作用下双层底的几个典型时刻等效塑性应变云图. (a) $t=0.2 \mathrm{~ms} ;$ (b) $t=1.5 \mathrm{~ms}$; (c) $t=$ $4.5 \mathrm{~ms}$; (d) $t=8 \mathrm{~ms}$

Figure 13 (Color online) Contour of equivalent plastic strain of the double bottom compartment subjected to contact underwater explosion at several typical times. (a) $t=0.2 \mathrm{~ms}$; (b) $t=1.5 \mathrm{~ms}$; (c) $t=4.5 \mathrm{~ms}$; (d) $t=8 \mathrm{~ms}$. 
(a)

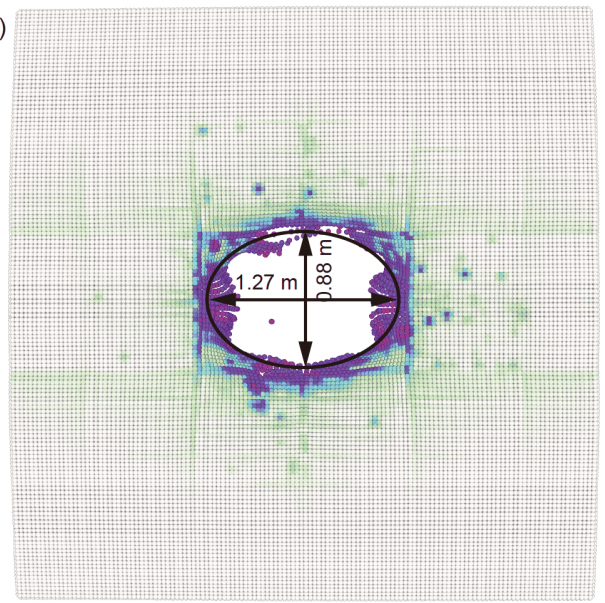

14 (网络版彩图)双层底水下接触爆炸SPH-RKPM与LS-DYNA/FEM计算结果对比. DYNA/FEM计算结果

Figure 14 (Color online) Comparison of SPH-RKPM and LS-DYNA/FEM results of the double bottom compartment subjected to underwater contact explosion. (a) SPH-RKPM result; (b) LS-DYNA/FEM result.

下, 舰船结构会产生严重毁伤, 通常情况下结构都会发 生大变形弹塑性断裂. 由于无网格算法不依赖拓扑结 构，其在结构断裂以及多相流模拟当中具有一定优势. 本文在文献 $[12,43]$ 的基础上，为了进一步地验证SPH 和RKPM耦合模型以及计算方法的有效性，建立了强 冲击载荷作用下舰船等复杂结构毁伤的完全无网格三 维模型与计算方法，并采用该计算模型对多个舰船结 构冲击毁伤问题进行数值模拟. 将本文计算结果与有 限元结果进行对比分析，结果表明采用SPH-RKPM计 算得到的结果和有限元结果吻合较好, 更加充分地验 证了本文计算模型、数值方法的有效性和正确性. (b)

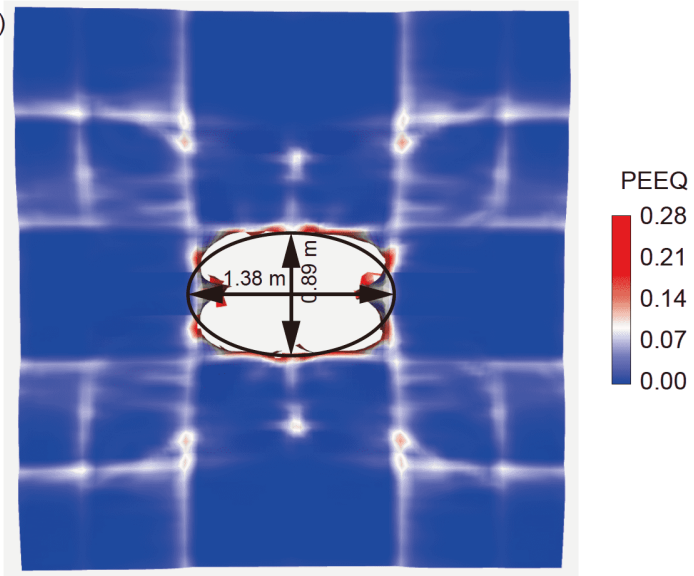

(a) SPH-RKPM计算结果; (b) LS-

(b) 
480-494

5 Jiang X J, Zhang P, Wu W F. Effect of impact position and initial velocity on side structure of the collided ship (in Chinese). Navig China, 2012, 35: 76-80 [姜兴家, 张鹏, 吴文锋. 撞击位置和初速度对被撞船舶舷侧结构的影响. 中国航海, 2012, 35: 76-80]

6 Zhang Z H, Wang Y. "Blade effect" on the overall damage of hull girder caused by near field undex shock wave (in Chinese). Sci Sin-Phys Mech Astron, 2015, 45: 054701 [张振华, 汪玉. 近距水爆冲击波对船体梁总体毁伤的“刀锋效应”. 中国科学: 物理学 力学 天文学, 2015, 45: 054701]

7 LeBlanc J M. Response of Composite Materials to Dynamic and Low Temperature Environments. Dissertation for Doctoral Degree. Kingston: University of Rhode Island, 2019

8 Wang X, Zhang S, Wang C, et al. Blast-induced damage and evaluation method of concrete gravity dam subjected to near-field underwater explosion. Eng Struct, 2020, 209: 109996

9 Rajendran R, Narasimhan K. Damage prediction of clamped circular plates subjected to contact underwater explosion. Int J Impact Eng, 2001, 25: 373-386

10 Zhang X C. Similarity criteria for experiment of underwater explosion (in Chinese). J Ship Mech, 2007, 11: 108-118 [张效慈. 水下爆炸试验相 似准则. 船舶力学, 2007, 11: 108-118]

11 Li S, Zhang A M, Han R. The mechanism of jetting behaviors of an oscillating bubble (in Chinese). Theor Appl Mech, 2019, 51: 1666-1681 [李 帅, 张阿漫, 韩荵. 水中高压脉动气泡水射流形成机理及载荷特性研究. 力学学报, 2019, 51: 1666-1681]

12 Peng Y X, Zhang A M, Ming F R. Numerical simulation of structural damage subjected to the near-field underwater explosion based on SPH and RKPM. Ocean Eng, 2021, 222: 108576

13 Zienkiewicz O C, Taylor R L, Taylor R L, et al. The Finite Element Method: Solid Mechanics. Oxford: Butterworth-Heinemann, 2000

14 Versteeg H K, Malalasekera W. An Introduction to Computational Fluid Dynamics: The Finite Volume Method. New York: Pearson Education, 2007

15 Rashidi S, Akar S, Bovand M, et al. Volume of fluid model to simulate the nanofluid flow and entropy generation in a single slope solar still. Renew Energy, 2018, 115: 400-410

16 Mirjalili S, Ivey C B, Mani A. A conservative diffuse interface method for two-phase flows with provable boundedness properties. J Comput Phys, 2020, 401: 109006, arXiv: 1803.01262

17 He M, Zhang A M, Liu Y L. Interaction of the underwater explosion bubbles and nearby double-layer structures with circular holes (in Chinese). Explos Shock Waves, 2020, 40: 40-50 [贺铭, 张阿漫, 刘云龙. 近场水下爆炸气泡与双层破口结构的相互作用. 爆炸与冲击, 2020, 40: 4050]

18 Zhang X, Song K Z, Lu M W. Research progress and application of meshless method (in Chinese). Chin J Comput Mech, 2003, 20: 730-742 [张 雄, 宋康祖, 陆明万. 无网格法研究进展及其应用. 计算力学学报, 2003, 20: 730-742]

19 Liu M, Zhang Z. Smoothed particle hydrodynamics (SPH) for modeling fluid-structure interactions. Sci China-Phys Mech Astron, 2019, 62: 984701

20 Monaghan J J. Simulating free surface flows with SPH. J Comput Phys, 1994, 110: 399-406

21 Becker M, Teschner M. Weakly compressible SPH for free surface flows. In: Proceedings of the 2007 ACM SIGGRAPH/Eurographics Symposium on Computer Animation, 2007. 209-217

22 Zhang A, Yang W S, Huang C, et al. Numerical simulation of column charge underwater explosion based on SPH and BEM combination. Comput Fluids, 2013, 71: 169-178

23 Liu M B, Liu G R, Lam K Y, et al. Smoothed particle hydrodynamics for numerical simulation of underwater explosion. Comput Mech, 2003, 30: $106-118$

24 Wang P P, Zhang A M, Meng Z F. An improved particle shifting algorithm for multiphase flows in SPH method (in Chinese). Chin Sci Bull, 2020, 65: 729-739 [王平平, 张阿漫, 孟子飞. 一种改进的适用于多相流SPH模拟的粒子位移修正算法. 科学通报, 2020, 65: 729-739]

25 Oger G, Doring M, Alessandrini B, et al. Two-dimensional SPH simulations of wedge water entries. J Comput Phys, 2006, 213: 803-822

26 Shao S. Incompressible SPH simulation of water entry of a free-falling object. Int J Numer Meth Fluids, 2009, 59: 91-115

27 De Leffe M, Le Touzé D, Alessandrini B. Normal flux method at the boundary for SPH. In: Proceedings of the 4th SPH Research and Engineering International Community Workshop, 2009

28 Liu W K, Jun S, Zhang Y F. Reproducing kernel particle methods. Int J Numer Methods Fluids, 1995, 20: 1081-1106 
29 Chen J S, Pan C, Wu C T, et al. Reproducing kernel particle methods for large deformation analysis of non-linear structures. Comput Methods Appl Mech Eng, 1996, 139: 195-227

30 Li S, Simonsen C B. Meshfree simulations of ductile crack propagations. Int J Comput Methods Eng Sci Mech, 2005, 6: 1-19

31 Ren B, Li S. Modeling and simulation of large-scale ductile fracture in plates and shells. Int J Solids Struct, 2012, 49: 2373-2393

32 Peng Y X. Improved Meshfree Methods and Its Application in Structural Damage Analysis Under Fluid-Structure Impact (in Chinese). Dissertation for Doctoral Degree. Harbin: Harbin Engineering University, 2019 [彭玉祥. 改进的无网格计算方法及其在结构流固耦合冲击毁 伤中的应用研究. 博士学位论文. 哈尔滨: 哈尔滨工程大学, 2019]

33 Belytschko T, Liu W K, Moran B, et al. Nonlinear Finite Elements for Continua and Structures. New Jersey: John Wiley \& Sons, 2013

34 Peng Y X, Zhang A M, Ming F R. A 3D meshfree crack propagation algorithm for the dynamic fracture in arbitrary curved shell. Comput Methods Appl Mech Eng, 2020, 367: 113139

35 Hughes T J R, Winget J. Finite rotation effects in numerical integration of rate constitutive equations arising in large-deformation analysis. Int J Numer Meth Eng, 1980, 15: 1862-1867

36 Lemaitre J, Chaboche J L. Mechanics of Solid Materials. Cambridge: Cambridge University Press, 1994

37 Moës N, Belytschko T. Extended finite element method for cohesive crack growth. Eng Fract Mech, 2002, 69: 813-833

38 Miehe C, Hofacker M, Welschinger F. A phase field model for rate-independent crack propagation: Robust algorithmic implementation based on operator splits. Comput Methods Appl Mech Eng, 2010, 199: 2765-2778

39 Nguyen T T, Waldmann D, Bui T Q. Role of interfacial transition zone in phase field modeling of fracture in layered heterogeneous structures. J Comput Phys, 2019, 386: 585-610

40 Belytschko T, Lu Y Y, Gu L. Crack propagation by element-free Galerkin methods. Eng Fract Mech, 1995, 51: 295-315

41 Kong X Z, Fang Q. Numerical predictions of failures in concrete structures subjected to intense dynamic loadings using the smooth particle hydrodynamics method (in Chinese). Sci Sin-Phys Mech Astron, 2020, 50: 024605 [孔祥振, 方秦. 基于SPH方法对强动载下混凝土结构损伤 破坏的数值模拟研究. 中国科学: 物理学 力学 天文学, 2020, 50: 024605]

42 Sakong J, Woo S C, Kim T W. Determination of impact fragments from particle analysis via smoothed particle hydrodynamics and $k$-means clustering. Int J Impact Eng, 2019, 134: 103387

43 Peng Y X, Zhang A M, Ming F R, et al. A meshfree framework for the numerical simulation of elasto-plasticity deformation of ship structure. Ocean Eng, 2019, 192: 106507 Geers T L, Hunter K S. An integrated wave-effects model for an underwater explosion bubble. J Acoust Soc Am, 2002, 111: 1584-1601 Zhao Y P. Suggestion of a new dimensionless number for dynamic plastic response of beams and plates. Arch Appl Mech, 1998, 68: 524-538 Johnson W, Sengupta A K, Ghosh S K, et al. Mechanics of high speed impact at normal incidence between plasticine long rods and plates. J Mech Phys Solids, 1981, 29: 413-445

47 Li Y, Wu W G, Wang Y, et al. Impact resistance of ship-build steel 945 and improved cowper-symonds models (in Chinese). Shipbuild China, 2014, 3: 94-100 [李营, 吴卫国, 汪玉, 等. 基于修正CS模型的船用945钢冲击性能研究. 中国造船, 2014, 3: 94-100] 


\title{
Numerical investigation of ship structure damage subject to strong impact using a 3D meshless SPH-RKPM method
}

\author{
PENG YuXiang, ZHANG AMan", XUE Bing \& LI ShaoBo \\ College of Shipbuilding Engineering, Harbin Engineering University, Harbin 150001, China
}

\begin{abstract}
The strong impact load will seriously threaten the ship vitality. The accurate prediction of the damage of ship structure under impact is an important step to the design of the ship protection structure to improve the ship vitality. Since the mesh-based method faces challenges in the simulation of structural fracture, on the basis of previous work, a complete meshless square model and calculation method of structural damage under strong impact load are established in this work. The SPH method is adopted in the calculation of fluid load in underwater explosion, and the RKPM is applied in the modeling of contact and fracture of the ship structure. Finally, based on the normal flux method and coupled with SPH-RKPM, a completely 3D meshless fluid-structure coupling numerical model is built, and several numerical complex examples verify the effectiveness of the numerical model. The numerical program for ship structural damage under strong impact is further developed and the robustness of the program is improved. As a module, the program has been integrated into the FSLAB industrial software with independent intellectual property rights. Compared with the traditional grid method, the numerical model has advantages in dealing with structural fracture and damage, and can provide necessary technical support for the design of ship protection structure.
\end{abstract}

structure damage, strong impact, smoothed particle hydrodynamics (SPH), reproducing kernel particle method (RKPM)

PACS: $47.40 . R s, 07.05 . \mathrm{Tp}$, 79.20.Ap, 61.20.Ja, 46.70.-p, 62.50.Ef

doi: $10.1360 /$ SSPMA-2020-0403 